\title{
LINEAR WEINGARTEN HYPERSURFACES WITH BOUNDED MEAN CURVATURE IN THE HYPERBOLIC SPACE
}

\author{
CÍCERO P. AQUINO \\ Departamento de Matemática, Universidade Federal do Piauí, \\ 64.049-550 Teresina, Piauí, Brazil \\ E-mail: cicero.aquino@ufpi.edu.br \\ HENRIQUE F. DE LIMA \\ Departamento de Matemática, Universidade Federal de Campina Grande, \\ 58.429-970 Campina Grande, Paraíba, Brazil \\ E-mail: henrique@dme.ufcg.edu.br \\ and MARCO ANTONIO L. VELÁSQUEZ \\ Departamento de Matemática, Universidade Federal de Campina Grande, \\ 58.429-970 Campina Grande, Paraíba, Brazil \\ E-mail: marco.velasquez@pq.cnpq.br
}

(Received 4 December 2013; accepted 13 March 2014; first published online 17 December 2014)

To Professor J. L. M. Barbosa, on occasion of his seventieth birthday

\begin{abstract}
We apply appropriate maximum principles in order to obtain characterization results concerning complete linear Weingarten hypersurfaces with bounded mean curvature in the hyperbolic space. By supposing a suitable restriction on the norm of the traceless part of the second fundamental form, we show that such a hypersurface must be either totally umbilical or isometric to a hyperbolic cylinder, when its scalar curvature is positive, or to a spherical cylinder, when its scalar curvature is negative. Related to the compact case, we also establish a rigidity result.
\end{abstract}

2000 Mathematics Subject Classification. Primary 53C42; Secondary 53C40.

1. Introduction and statemeof the main results. In the theory of isometric immersions, the study of complete hypersurfaces with constant scalar curvature immersed in a Riemannian space constitutes an important theme. In the seminal paper [10], Cheng and Yau introduced a new self-adjoint differential operator $\square$ acting on smooth functions defined on Riemannian manifolds. As a by-product of such approach they were able to classify closed hypersurfaces with constant normalized scalar curvature $R$ satisfying $R \geq c$ and nonnegative sectional curvature immersed in a real space form $\mathbb{Q}_{c}^{n+1}$ of constant sectional curvature $c$. Later on, Li [11] extended the results due to Cheng and Yau [10] in terms of the squared norm of the second fundamental form of the hypersurface.

In [18], Shu applied the so-called generalized maximum principle of Omori-Yau $[15,20]$ to prove that a complete hypersurface in the hyperbolic space $\mathbb{H}^{n+1}$ with constant normalized scalar curvature and nonnegative sectional curvature must be either totally umbilical or isometric to a hyperbolic cylinder of $\mathbb{W}^{n+1}$. Afterwards, 
Brasil Jr., Colares and Palmas [7] also used the generalized maximum principle of Omori-Yau to characterize complete hypersurfaces with constant scalar curvature in the unit Euclidean sphere $\mathbb{S}^{n+1}$. In [2], by applying a weak Omori-Yau maximum principle due to Pigola, Rigoli and Setti [16], Alías and García-Martínez studied the behaviour of the scalar curvature $R$ of a complete hypersurface immersed with constant mean curvature into $\mathbb{Q}_{c}^{n+1}$, deriving a sharp estimate for the infimum of $R$. Next, Alías, García-Martínez and Rigoli [3] obtained another suitable weak maximum principle for complete hypersurfaces with constant scalar curvature in $\mathbb{Q}_{c}^{n+1}$, and gave some applications of it in order to estimate the norm of the traceless part $\Phi$ of the second fundamental form. In particular, they extended the main theorem of [7] for the context of $\mathbb{Q}_{c}^{n+1}$.

In [12], Li, Suh and Wei studied linear Weingarten hypersurfaces immersed in $\mathbb{S}^{n+1}$, that is, hypersurfaces whose mean curvature $H$ and normalized scalar curvature $R$ satisfy $R=a H+b$, for some $a, b \in \mathbb{R}$. In this setting, they showed that if $M^{n}$ is a compact linear Weingarten hypersurface with nonnegative sectional curvature immersed in $\mathbb{S}^{n+1}$, such that $R=a H+b$ with $(n-1) a^{2}+4 n(b-1) \geq 0$, then $M^{n}$ is either totally umbilical or isometric to a Clifford torus $\mathbb{S}^{k}\left(c_{1}\right) \times \mathbb{S}^{n-k}\left(c_{2}\right)$, where $1 \leq k \leq n-1, c_{1}, c_{2}>0$ and $\frac{1}{c_{1}}+\frac{1}{c_{2}}=1$. Thereafter, Shu [19] proved another rigidity theorems concerning linear Weingarten hypersurfaces with two distinct principal curvatures immersed in a space form $\mathbb{Q}_{c}^{n+1}$. We also point out that López [13], and Barros, Silva and Sousa $[\mathbf{5 , 6}$ ] obtained descriptions related to rotational linear Weingarten surfaces in the Euclidean space and in the Euclidean sphere, respectively.

Furthermore, the first and second authors [4] used the Hopf's strong maximum principle and an extension of a suitable maximum principle of Yau [21] due to Caminha [8] in order to study the geometry of complete linear Weingarten hypersurfaces with nonnegative sectional curvature immersed in the hyperbolic space $\mathbb{Q}^{n+1}$. In this setting, under appropriated restrictions on the mean curvature attains its maximum, they proved that such a hypersurface must be either totally umbilical or isometric to a hyperbolic cylinder $\mathbb{U}^{1}\left(c_{1}\right) \times \mathbb{S}^{n-1}\left(c_{2}\right)$, where $c_{1}<0, c_{2}>0$ and $\frac{1}{c_{1}}+\frac{1}{c_{2}}=-1$.

Motivated by the works described above, our aim is to establish new characterizations of complete linear Weingarten hypersurfaces immersed in $\mathbb{\boxplus}^{n+1}$. In this setting, we apply an extension of the generalized maximum principle of Omori jointly with Hopf's strong maximum principle to an appropriated modified Cheng-Yau operator in order to prove that such a hypersurface must be either totally umbilical or isometric to a hyperbolic cylinder, when its scalar curvature is positive, or to a spherical cylinder, when its scalar curvature is negative. More precisely, we obtain the following classification result:

THEOREM 1.1. Let $M^{n}$ be a complete linear Weingarten hypersurface immersed in $\mathbb{M}^{n+1}$, such that $R=a H+b$ with $a \leq 0$ and $(n-1) a^{2}+4 n(b+1) \geq 0$. Suppose that $1 \leq H^{2} \leq \alpha$, for some constant $\alpha$. If

$$
\sup |\Phi| \leq \mathcal{R}_{H}^{+},
$$

where

$$
\mathcal{R}_{H}^{+}=\frac{1}{2} \sqrt{\frac{n}{n-1}}\left(\sqrt{n^{2} H^{2}-4(n-1)}-(n-2) H\right),
$$

then 
(i) either $|\Phi| \equiv 0$ and $M^{n}$ is totally umbilical,

(ii) or $\sup |\Phi|=\mathcal{R}_{H}^{+}$. In addition, if $b>-1$ and $|\Phi(p)|=\mathcal{R}_{H}^{+}$at some point $p \in M^{n}$, then $|\Phi| \equiv \mathcal{R}_{H}^{+}$and $M^{n}$ is isometric to a hyperbolic cylinder $\mathbb{H}^{1}\left(-\sqrt{1+r^{2}}\right) \times$ $\mathbb{S}^{n-1}(r)$, with $R=\frac{n-2}{n r^{2}}>0$, or to a spherical cylinder $\mathbb{H}^{n-1}\left(-\sqrt{1+r^{2}}\right) \times \mathbb{S}^{1}(r)$, with $R=-\frac{n-2}{n\left(1+r^{2}\right)}<0$.

Related to the compact case, we also get the following rigidity result:

THEOREM 1.2. Let $M^{n}$ be a compact linear Weingarten hypersurface immersed in $\mathbb{H}^{n+1}$, such that $R=a H+b$ with $(n-1) a^{2}+4 n(b+1) \geq 0$. Suppose that $H^{2} \geq 1$. If $\sup |\Phi|<\mathcal{R}_{H}^{+}$, where $\mathcal{R}_{H}^{+}=\frac{1}{2} \sqrt{\frac{n}{n-1}}\left(\sqrt{n^{2} H^{2}-4(n-1)}-(n-2) H\right)$, then $|\Phi| \equiv 0$ and $M^{n}$ is isometric to $\mathbb{S}^{n}$, up to scaling.

The proofs of Theorems 1.1 and 1.2 are given in Section 4.

2. Preliminaries. Let $M^{n}$ be an $n$-dimensional, connect and orientable hypersurface in $\mathbb{H}^{n+1}$. We choose a local field of orthonormal frame $\left\{e_{A}\right\}_{1 \leq A \leq n+1}$ in $\mathbb{H}^{n+1}$, with dual coframe $\left\{\omega_{A}\right\}_{1 \leq A \leq n+1}$, such that, at each point of $M^{n}, e_{1}, \ldots, e_{n}$ are tangent to $M^{n}$ and $e_{n+1}$ is normal to $M^{n}$. We will use the following convention for the indices:

$$
1 \leq A, B, C, \ldots, \leq n+1,1 \leq i, j, k, \ldots, \leq n
$$

Denoting by $\left\{\omega_{A B}\right\}$ the connection forms of $\mathbb{H}^{n+1}$, we have that the structure equations of $\mathbb{W}^{n+1}$ are given by:

$$
\begin{aligned}
d \omega_{A} & =\sum_{i} \omega_{A i} \wedge \omega_{i}+\omega_{A n+1} \wedge \omega_{n+1}, \quad \omega_{A B}+\omega_{B A}=0, \\
d \omega_{A B} & =\sum_{C} \varepsilon_{C} \omega_{A C} \wedge \omega_{C B}-\frac{1}{2} \sum_{C, D} K_{A B C D} \omega_{C} \wedge \omega_{D}, \\
K_{A B C D} & =-\left(\delta_{A C} \delta_{B D}-\delta_{A D} \delta_{B C}\right) .
\end{aligned}
$$

Next, we restrict all the tensors to $M^{n}$. First of all, $\omega_{n+1}=0$ on $M^{n}$, so $\sum_{i} \omega_{n+1 i} \wedge$ $\omega_{i}=d \omega_{n+1}=0$ and by Cartan's lemma [9] we can write

$$
\omega_{n+1 i}=\sum_{j} h_{i j} \omega_{j}, h_{i j}=h_{j i} .
$$

This gives the second fundamental form of $M^{n}, B=\sum_{i j} h_{i j} \omega_{i} \omega_{j} e_{n+1}$. Furthermore, the mean curvature $H$ of $M^{n}$ is defined by $H=\frac{1}{n} \sum_{i} h_{i i}$.

The structure equations of $M^{n}$ are given by

$$
\begin{aligned}
& d \omega_{i}=\sum_{j} \omega_{i j} \wedge \omega_{j}, \quad \omega_{i j}+\omega_{j i}=0, \\
& d \omega_{i j}=\sum_{k} \omega_{i k} \wedge \omega_{k j}-\frac{1}{2} \sum_{k, l} R_{i j k l} \omega_{k} \wedge \omega_{l} .
\end{aligned}
$$


Using the structure equations, we obtain the Gauss equation

$$
R_{i j k l}=-\left(\delta_{i k} \delta_{j l}-\delta_{i l} \delta_{j k}\right)+\left(h_{i k} h_{j l}-h_{i l} h_{j k}\right),
$$

where $R_{i j k l}$ are the components of the curvature tensor of $M^{n}$.

The Ricci curvature and the normalized scalar curvature of $M^{n}$ are given, respectively, by

$$
R_{i j}=-(n-1) \delta_{i j}+n H h_{i j}-\sum_{k} h_{i k} h_{k j}
$$

and

$$
R=\frac{1}{n(n-1)} \sum_{i} R_{i i}
$$

From (2.8) and (2.9) we obtain

$$
|B|^{2}=n^{2} H^{2}-n(n-1)(R+1),
$$

where $|B|^{2}=\sum_{i, j} h_{i j}^{2}$ is the square of the length of the second fundamental form $B$ of $M^{n}$.

The components $h_{i j k}$ of the covariant derivative $\nabla B$ satisfy

$$
\sum_{k} h_{i j k} \omega_{k}=d h_{i j}+\sum_{k} h_{i k} \omega_{k j}+\sum_{k} h_{j k} \omega_{k i} .
$$

The Codazzi equation and the Ricci identity are, respectively, given by

$$
h_{i j k}=h_{i k j}
$$

and

$$
h_{i j k l}-h_{i j l k}=\sum_{m} h_{m j} R_{m i k l}+\sum_{m} h_{i m} R_{m j k l},
$$

where $h_{i j k}$ and $h_{i j k}$ denote the first and the second covariant derivatives of $h_{i j}$.

The Laplacian $\Delta h_{i j}$ of $h_{i j}$ is defined by $\Delta h_{i j}=\sum_{k} h_{i j k k}$. From equations (2.12) and (2.13), we obtain that

$$
\Delta h_{i j}=\sum_{k} h_{k k i j}+\sum_{k, l} h_{k l} R_{l j k k}+\sum_{k, l} h_{l i} R_{l l j k} .
$$

Since $\Delta|B|^{2}=2\left(\sum_{i, j} h_{i j} \Delta h_{i j}+\sum_{i, j, k} h_{i j k}^{2}\right)$, from (2.14) we get

$$
\begin{aligned}
\frac{1}{2} \Delta|B|^{2}= & |\nabla B|^{2}+\sum_{i, i, k} h_{i j} h_{k k i j}+\sum_{i, j, k, l} h_{i j} h_{l k} R_{l i j k} \\
& +\sum_{i, j, k, l} h_{i j} h_{i l} R_{l k j k} .
\end{aligned}
$$


Consequently, taking a (local) orthonormal frame $\left\{e_{1}, \ldots, e_{n}\right\}$ on $M^{n}$ such that $h_{i j}=\lambda_{i} \delta_{i j}$, from equation (2.15) we obtain the following Simons-type formula

$$
\frac{1}{2} \Delta|B|^{2}=|\nabla B|^{2}+\sum_{i} \lambda_{i}(n H)_{, i i}+\frac{1}{2} \sum_{i, j} R_{i j i j}\left(\lambda_{i}-\lambda_{j}\right)^{2} .
$$

Now, let $\phi=\sum_{i, j} \phi_{i j} \omega_{i} \omega_{j}$ be a symmetric tensor on $M^{n}$ defined by

$$
\phi_{i j}=n H \delta_{i j}-h_{i j} .
$$

Following Cheng-Yau [10], we introduce an operator $\square$ associated to $\phi$ acting on any smooth function $f$ by

$$
\square f=\sum_{i, j} \phi_{i j} f_{i j}=\sum_{i, j}\left(n H \delta_{i j}-h_{i j}\right) f_{i j} .
$$

Since $\phi_{i j}$ is divergence-free, it also follows from [10] that the operator $\square$ is selfadjoint relative to the $L^{2}$ inner product of $M^{n}$, that is,

$$
\int_{M} f \square g=\int_{M} g \square f,
$$

for any smooth functions $f$ and $g$ on $M^{n}$.

Setting $f=n H$ in (2.17) and taking a local frame field $\left\{e_{1}, \ldots, e_{n}\right\}$ on $M^{n}$ such that $h_{i j}=\lambda_{i} \delta_{i j}$, from equation (2.10) we obtain the following:

$$
\begin{aligned}
\square(n H) & =n H \Delta(n H)-\sum_{i} \lambda_{i}(n H)_{, i i} \\
& =\frac{1}{2} \Delta(n H)^{2}-\sum_{i}(n H)_{, i}^{2}-\sum_{i} \lambda_{i}(n H)_{, i i} \\
& =\frac{n(n-1)}{2} \Delta R+\frac{1}{2} \Delta|B|^{2}-n^{2}|\nabla H|^{2}-\sum_{i} \lambda_{i}(n H)_{, i i} .
\end{aligned}
$$

Consequently, taking into account equation (2.16), we get

$$
\square(n H)=\frac{n(n-1)}{2} \Delta R+|\nabla B|^{2}-n^{2}|\nabla H|^{2}+\frac{1}{2} \sum_{i, j} R_{i j i j}\left(\lambda_{i}-\lambda_{j}\right)^{2} .
$$

3. Key lemmas. Along this section, we will quote some key lemmas which we will use in the proofs of Theorems 1.1 and 1.2. The first one is a classic algebraic result due to Okumura [14], and completed with the equality case proved in [1] by Alencar and do Carmo.

LEMMA 3.1. Let $\mu_{1}, \ldots, \mu_{n}$ be real numbers such that $\sum_{i} \mu_{i}=0$ and $\sum_{i} \mu_{i}^{2}=\beta^{2}$, with $\beta \geq 0$. Then,

$$
-\frac{(n-2)}{\sqrt{n(n-1)}} \beta^{3} \leq \sum_{i} \mu_{i}^{3} \leq \frac{(n-2)}{\sqrt{n(n-1)}} \beta^{3}
$$


and equality holds if, and only if, either at least $(n-1)$ of the numbers $\mu_{i}$ are equal.

The second lemma is obtained reasoning as in the proof of Lemma 2.1 of [12] (see Lemma 3.1 of [4]).

LEMMA 3.2. Let $M^{n}$ be a linear Weingarten hypersurface immersed in $\mathbb{H}^{n+1}$, such that $R=a H+b$ for some $a, b \in \mathbb{R}$. Suppose that

$$
(n-1) a^{2}+4 n(b+1) \geq 0 .
$$

Then

$$
|\nabla B|^{2} \geq n^{2}|\nabla H|^{2} .
$$

Moreover, if the inequality (3.20) is strict and the equality holds in (3.21) on $M^{n}$, then $H$ is constant on $M^{n}$.

Now, we will consider the following Cheng-Yau's modified operator

$$
L=\square-\frac{n-1}{2} a \Delta .
$$

Related to such operator, we have the following sufficient criteria of ellipticity (see Lemma 3.2 of [4]).

LEMMA 3.3. Let $M^{n}$ be a linear Weingarten hypersurface immersed in $\mathbb{1}^{n+1}$, such that $R=a H+b$ with $b>-1$. Then, $L$ is elliptic.

In our last lemma, we extend the generalized maximum principle of Omori [15] to the Cheng-Yau's modified operator $L$.

LEMMA 3.4. Let $M^{n}$ be a complete linear Weingarten hypersurface immersed in $\mathbb{H}^{n+1}$, such that $R=a H+b$ with $a \leq 0$ and $(n-1) a^{2}+4 n(b+1) \geq 0$. If $H$ is bounded on $M^{n}$, then there exists a sequence of points $\left\{p_{k}\right\}_{k \geq 1}$ in $M^{n}$ satisfying the following properties:

$$
\lim _{k \rightarrow \infty} n H\left(p_{k}\right)=n \sup H, \lim _{k \rightarrow \infty}\left|\nabla n H\left(p_{k}\right)\right|=0 \text { and } \limsup _{k \rightarrow \infty}\left(L(n H)\left(p_{k}\right)\right) \leq 0 .
$$

Proof. Let us choose a local orthonormal frame $\left\{e_{1}, \ldots, e_{n}\right\}$ on $M^{n}$ such that $h_{i j}=\lambda_{i} \delta_{i j}$. From (3.22) we have that

$$
L(n H)=\sum_{i}\left(n H-\frac{n-1}{2} a-\lambda_{i}\right)(n H)_{i i} .
$$

On the other hand, we observe that, if $H$ vanishes identically on $M^{n}$, the lemma is obvious. So, let us suppose that $H$ is not identically zero. By changing the orientation of $M^{n}$ if necessary, we may assume $\sup H>0$. Thus, for all $i=1, \ldots, n$, from (2.10) and with a straightforward computation we get

$$
\begin{aligned}
\left(\lambda_{i}\right)^{2} & \leq|B|^{2}=n^{2} H^{2}-n(n-1)(a H+b+1) \\
& =\left(n H-\frac{n-1}{2} a\right)^{2}-\frac{n-1}{4}\left((n-1) a^{2}+4 n(b+1)\right) \\
& \leq\left(n H-\frac{n-1}{2} a\right)^{2},
\end{aligned}
$$


where we have used our assumption that $(n-1) a^{2}+4 n(b+1) \geq 0$ to obtain the last inequality. Consequently, for all $i=1, \ldots, n$, we have

$$
\left|\lambda_{i}\right| \leq\left|n H-\frac{n-1}{2} a\right|
$$

Thus, from (2.7) and (3.24) we obtain

$$
R_{\ddot{i} \ddot{j}}=-1+\lambda_{i} \lambda_{j} \geq-1-\left(n H-\frac{n-1}{2} a\right)^{2} .
$$

Hence, since we are supposing that $H$ is bounded on $M^{n}$, it follows from (3.25) that the sectional curvatures of $M^{n}$ are bounded from below. Therefore, we can apply the generalized maximum principle due to Omori [15] to the function $n H$, obtaining a sequence of points $\left\{p_{k}\right\}_{k \geq 1}$ in $M^{n}$ such that

$$
\lim _{k \rightarrow \infty} n H\left(p_{k}\right)=n \sup H, \lim _{k \rightarrow \infty}\left|\nabla n H\left(p_{k}\right)\right|=0, \limsup _{k \rightarrow \infty}\left((n H)_{i i}\left(p_{k}\right)\right) \leq 0 .
$$

But, since $H$ is bounded, taking subsequences if necessary, we can arrive to a sequence $\left\{p_{k}\right\}_{k \geq 1}$ in $M^{n}$ which satisfies (3.26) and such that $H\left(p_{k}\right) \geq 0$. Thus, taking into account that $a \leq 0$, from (3.24) we get

$$
\begin{aligned}
0 & \leq n H\left(p_{k}\right)-\frac{n-1}{2} a-\left|\lambda_{i}\left(p_{k}\right)\right| \leq n H\left(p_{k}\right)-\frac{n-1}{2} a-\lambda_{i}\left(p_{k}\right) \\
& \leq n H\left(p_{k}\right)-\frac{n-1}{2} a+\left|\lambda_{i}\left(p_{k}\right)\right| \leq 2 n H\left(p_{k}\right)-(n-1) a .
\end{aligned}
$$

Consequently, using once more that $H$ is bounded on $M^{n}$, from (3.27) we infer that $n H\left(p_{k}\right)-\frac{n-1}{2} a-\lambda_{i}\left(p_{k}\right)$ is nonnegative and bounded on $M^{n}$. Therefore, from (3.23), (3.26) and (3.27), we obtain that

$$
\limsup _{k \rightarrow \infty}\left(L(n H)\left(p_{k}\right)\right) \leq \sum_{i} \limsup _{k \rightarrow \infty}\left[\left(n H-\frac{n-1}{2} a-\lambda_{i}\right)\left(p_{k}\right)(n H)_{i i}\left(p_{k}\right)\right] \leq 0 .
$$

4. Proofs of Theorems 1.1 and 1.2. Let us start with the proof of Theorem 1.1.

Proof.

Let us choose a local orthonormal frame $\left\{e_{1}, \ldots, e_{n}\right\}$ on $M^{n}$ such that $h_{i j}=\lambda_{i} \delta_{i j}$. Since $R=a H+b$, from (2.18) and (3.22) we have that

$$
L(n H)=|\nabla B|^{2}-n^{2}|\nabla H|^{2}+\frac{1}{2} \sum_{i, j} R_{i j i j}\left(\lambda_{i}-\lambda_{j}\right)^{2} .
$$

Thus, since from (2.7) we have that $R_{\ddot{j} \dddot{j}}=\lambda_{i} \lambda_{j}-1$, from (4.28) we get

$$
L(n H)=|\nabla B|^{2}-n^{2}|\nabla H|^{2}+n^{2} H^{2}-n|B|^{2}-|B|^{4}+n H \sum_{i} \lambda_{i}^{3} .
$$


Now, set $\Phi_{i j}=h_{i j}-H \delta_{i j}$. We will consider the following symmetric tensor

$$
\Phi=\sum_{i, j} \Phi_{i j} \omega_{i} \omega_{j}
$$

Let $|\Phi|^{2}=\sum_{i, j} \Phi_{i j}^{2}$ be the square of the length of $\Phi$. It is easy to check that $\Phi$ is traceless and

$$
|\Phi|^{2}=|B|^{2}-n H^{2}
$$

With respect the frame field $\left\{e_{1}, \ldots, e_{n}\right\}$ on $M^{n}$, we have that $\Phi_{i j}=\mu_{i} \delta_{i j}$ and, with a straightforward computation, we verify that

$$
\sum_{i} \mu_{i}=0, \sum_{i} \mu_{i}^{2}=|\Phi|^{2} \text { and } \sum_{i} \mu_{i}^{3}=\sum_{i} \lambda_{i}^{3}-3 H|\Phi|^{2}-n H^{3}
$$

Thus, using Gauss equation (2.7) jointly with (4.31) into (4.29), we get

$$
\begin{aligned}
L(n H)= & |\nabla B|^{2}-n^{2}|\nabla H|^{2}+n H \sum_{i} \mu_{i}^{3} \\
& +|\Phi|^{2}\left(-|\Phi|^{2}+n H^{2}-n\right) .
\end{aligned}
$$

By applying Lemmas 3.1 and 3.2, from (4.32) we have

$$
\begin{aligned}
L(n H) & \geq|\Phi|^{2}\left(-|\Phi|^{2}-\frac{n(n-2)}{\sqrt{n(n-1)}} H|\Phi|+n H^{2}-n\right) \\
& =|\Phi|^{2} P_{H}(|\Phi|),
\end{aligned}
$$

where

$$
P_{H}(|\Phi|)=-|\Phi|^{2}-\frac{n(n-2)}{\sqrt{n(n-1)}} H|\Phi|+n H^{2}-n .
$$

Since we are supposing that $H^{2} \geq 1$, from (4.34) it is easy to verify that $P_{H}(|\Phi|)$ has two real roots $\mathcal{R}_{H}^{-}$and $\mathcal{R}_{H}^{+}$given by

$$
\mathcal{R}_{H}^{-}=-\frac{1}{2} \sqrt{\frac{n}{n-1}}\left(\sqrt{n^{2} H^{2}-4(n-1)}+(n-2) H\right)
$$

and

$$
\mathcal{R}_{H}^{+}=\frac{1}{2} \sqrt{\frac{n}{n-1}}\left(\sqrt{n^{2} H^{2}-4(n-1)}-(n-2) H\right) .
$$

Consequently, we have that

$$
P_{H}(|\Phi|)=\left(|\Phi|-\mathcal{R}_{H}^{-}\right)\left(\mathcal{R}_{H}^{+}-|\Phi|\right) .
$$

Thus, since we are assuming that $|\Phi| \leq \mathcal{R}_{H}^{+}$, from (4.35) we conclude that $P_{H}(|\Phi|) \geq 0$. Hence, from (4.33) we get

$$
L(n H) \geq|\Phi|^{2} P_{H}(|\Phi|) \geq 0 .
$$


On the other hand, by Lemma 3.4 it is possible to obtain a sequence of points $\left\{p_{k}\right\}_{k \geq 1}$ in $M^{n}$ such that

$$
\lim _{k \rightarrow \infty} H\left(p_{k}\right)=\sup H>0 \text { and } \limsup _{k \rightarrow \infty}\left(L(n H)\left(p_{k}\right)\right) \leq 0 .
$$

Thus, since from (2.10) we have that

$$
|\Phi|^{2}=n(n-1)\left(H^{2}-a H-b-1\right),
$$

our assumption that $a \leq 0$ jointly with (4.37) give

$$
\lim _{k \rightarrow \infty}\left|\Phi\left(p_{k}\right)\right|=\sup |\Phi| .
$$

Consequently, from (4.36) and (4.39), we have that

$$
0 \geq \limsup _{k \rightarrow \infty}\left(L(n H)\left(p_{k}\right)\right) \geq \sup |\Phi|^{2} P_{\sup H}(|\sup \Phi|) \geq 0
$$

and, hence, we conclude that $\sup |\Phi|^{2} P_{\sup H}(|\sup \Phi|)=0$. Therefore, we have that either $|\Phi| \equiv 0$ and $M^{n}$ is totally umbilical or $\sup |\phi|=\mathcal{R}_{H}^{+}$. Moreover, if $|\Phi(p)|=$ $\mathcal{R}_{H}^{+}(p)$ at some point $p \in M^{n}$ and since we are assuming that $a \leq 0$, equation (4.38) implies that $H$ attains its maximum on $M^{n}$. Thus, since Lemma 3.3 guarantees that $L$ is elliptic when $b>-1$, from inequality (4.36) we can apply Hopf's strong maximum principle to conclude that $H$ is constant on $M^{n}$. Consequently, $|\Phi|=\mathcal{R}_{H}^{+}$on $M^{n}$ and, since the equality holds in (3.19) of Lemma 3.1, we conclude that $M^{n}$ must be an isoparametric hypersurface with two distinct principal curvatures one of which is simple. Therefore, in this case, from the classification of the complete isoparametric hypersurfaces having at most two distinct principal curvatures due to Ryan [17], we conclude that $M^{n}$ is isometric to a hyperbolic cylinder $\mathbb{S}^{n-1}(r) \times \mathbb{M}^{1}\left(-\sqrt{1+r^{2}}\right)$, if $R>0$, or to a spherical cylinder $\mathbb{S}^{1}(r) \times \mathbb{W}^{n-1}\left(-\sqrt{1+r^{2}}\right)$, if $R<0$.

To conclude the proof, we observe that, for a given radius $r>0$, the standard product embedding $\mathbb{W}^{1}\left(-\sqrt{1+r^{2}}\right) \times \mathbb{S}^{n-1}(r) \hookrightarrow \mathbb{H}^{n+1}$ has constant principal curvatures given by

$$
k_{1}=\frac{r}{\sqrt{1+r^{2}}}, \quad k_{2}=\cdots=k_{n}=\frac{\sqrt{1+r^{2}}}{r} .
$$

Thus, in this case,

$$
H=\frac{n r^{2}+(n-1)}{n r \sqrt{1+r^{2}}} \text { and }|\Phi|^{2}=\frac{n-1}{n r^{2}\left(1+r^{2}\right)} .
$$

Consequently, from (2.10) and (4.30), we obtain that

$$
R=\frac{n-2}{n r^{2}}>0
$$

On the other hand, the embedding $\mathbb{H}^{n-1}\left(-\sqrt{1+r^{2}}\right) \times \mathbb{S}^{1}(r) \hookrightarrow \mathbb{H}^{n+1}$ has constant principal curvatures given by

$$
k_{1}=\cdots=k_{n-1}=\frac{r}{\sqrt{1+r^{2}}}, \quad k_{n}=\frac{\sqrt{1+r^{2}}}{r} .
$$


In this another case, we have

$$
H=\frac{n r^{2}+(n-1)}{n r \sqrt{1+r^{2}}} \text { and }|\Phi|^{2}=\frac{n-1}{n r^{2}\left(1+r^{2}\right)} .
$$

Hence, using once more (2.10) and (4.30) we get that

$$
R=-\frac{n-2}{n\left(1+r^{2}\right)}<0 .
$$

Moreover, with algebraic computations we verify that in both of these previous cases we have $|\Phi| \equiv \mathcal{R}_{H}^{+}$.

We close our paper by presenting the proof of Theorem 1.2.

Proof.

Since the operator $L$ is self-adjoint relative to the $L^{2}$ inner product of the compact hypersurface $M^{n}$, from inequality (4.36) we have that

$$
0=\int_{M} L(n H) d M \geq \int_{M}|\Phi|^{2} P_{H}(|\Phi|) d M \geq 0 .
$$

Consequently, since we are supposing that $\sup |\Phi|<\mathcal{R}_{H}^{+}$, from (4.40) we get that $|\phi| \equiv 0$ on $M^{n}$. Therefore, $M^{n}$ is totally umbilical and, hence, taking into account again the compactness of $M^{n}$, from the classification of the totally umbilical hypersurfaces of $\mathfrak{H}^{n+1}$ we have that $M^{n}$ must be isometric to $\mathbb{S}^{n}(r)$, for some $r>0$.

ACKNOWLEDGEMENTS. The authors would like to thank the referee for giving some interesting comments. The second author is partially supported by CNPq, Brazil, grant 300769/2012-1. The second and third authors are partially supported by CAPES/CNPq, Brazil, grant Casadinho/Procad 552.464/2011-2.

\section{REFERENCES}

1. H. Alencar and M. do Carmo, Hypersurfaces with constant mean curvature in spheres, Proc. Am. Math. Soc. 120 (1994), 1223-1229.

2. L. J. Alías and S. C. García-Martínez, On the scalar curvature of constant mean curvature hypersurfaces in space forms, J. Math. Anal. Appl. 363 (2010), 579-587.

3. L. J. Alías, S. C. García-Martínez and M. Rigoli, A maximum principle for hypersurfaces with constant scalar curvature and applications, Ann. Glob. Anal. Geom. 41 (2012), 307-320.

4. C. P. Aquino and H. F. de Lima, On the geometry of linearWeingarten hypersurfaces in the hyperbolic space, Monatsh. Math. 171 (2013), 259-268.

5. A. Barros, J. Silva and P. Sousa, Rotational Linear Weingarten surfaces into the Euclidean sphere, Israel J. Math. 192 (2012), 819-830.

6. A. Barros, J. Silva and P. Sousa, Rotational linear Weingarten hypersurfaces into the Euclidean sphere $\mathbb{S}^{n+1}, A d v$. Geom., to appear.

7. A. Brasil Jr., A. G. Colares and O. Palmas, Complete hypersurfaces with constant scalar curvature in spheres, Monatsh. Math. 161 (2010), 369-380.

8. A. Caminha, The geometry of closed conformal vector fields on Riemannian spaces, Bull. Braz. Math. Soc. 42 (2011), 277-300.

9. É. Cartan, Familles de surfaces isoparamétriques dans les espaces à courbure constante, Ann. Mat. Pura Appl. 17 (1938), 177-191.

10. S. Y. Cheng and S. T. Yau, Hypersurfaces with constant scalar curvature, Math. Ann. 225 (1977), 195-204. 
11. H. Li, Hypersurfaces with constant scalar curvature in space forms, Math. Ann. 305 (1996), 665-672.

12. H. Li, Y. J. Suh and G. Wei, Linear Weingarten hypersurfaces in a unit sphere, Bull. Korean Math. Soc. 46 (2009), 321-329.

13. R. López, Rotational linear Weingarten surfaces of hyperbolic type, Israel J. Math. 167 (2008), 283-301.

14. M. Okumura, Hypersurfaces and a pinching problem on the second fundamental tensor, Am. J. Math. 96 (1974), 207-213.

15. H. Omori, Isometric immersions of Riemannian manifolds, J. Math. Soc. Japan 19 (1967), 205-214.

16. S. Pigola, M. Rigoli and A. G. Setti, Maximum principles on Riemannian manifolds and applications, Mem. Am. Math. Soc. 822 (2005), 1-95.

17. P. J. Ryan, Hypersurfaces with parallel Ricci tensor, Osaka J. Math. 8 (1971), 251-259.

18. S. Shu, Complete hypersurfaces with constant scalar curvature in a hyperbolic space, Balkan J. Geom. Appl. 12 (2007), 107-115.

19. S. Shu, Linear Weingarten hypersurfaces in a real space form, Glasgow Math. J. 52 (2010), 635-648.

20. S. T. Yau, Harmonic functions on complete Riemannian manifolds, Commun. Pure Appl. Math. 28 (1975), 201-228.

21. S.T. Yau, Some function-theoretic properties of complete Riemannian manifolds and their applications to geometry, Indiana Univ. Math. J. 25 (1976), 659-670. 